\title{
THE BEGINNINGS OF APPLIED MATHEMATICS AFTER THE SECOND WORLD WAR
}

\author{
BY \\ PETER D. LAX \\ New York University-Courant Institute
}

1. I bring fraternal greetings from the Courant Institute of New York University to the Applied Mathematics Division of Brown University. The two institutions are twins, not identical, to be sure. Both are creatures of wartime mathematical projects sponsored by the Office of Scientific Research and Development (ORSD); both had a stellar cast of characters. At NYU, Courant, Friedrichs, Stoker, Friedman, Lewy, Shiffman and Spencer; Brown had Tamarkin, Bers, Feller, Hurewicz, Loewner and Polya, augmented during summers by Friedrichs, von Mises, and Synge.

Today computing is an essential, integral and highly visible part of applied mathematics. Back in the forties the need for computing was just as great, but the means of accomplishing it much more modest. The mathematics group at Brown had the inestimable fortune to have access to Aiken's Mark II computer at Harvard. The time allotted to Brown was sometimes bootlegged to investigate questions of pure mathematics. Carl Loewner liked to describe the following instance.

One of the striking manifestations in the theory of Fourier series is the Gibbs phenomenon, the overshoot of the partial sums of the Fourier series of a discontinuous function. On the other hand, Fejér has shown that the arithmetic means of the partial sums display no such overshoot; this is a consequence of positivity of the kernel expressing the arithmetic means in terms of the function. Cesàro has defined, in a natural way, arithmetic means of any order of a sequence, even of fractional order. When applied to the partial sums of a Fourier series, the kernel of the arithmetic means of order $c$ is positive for $c \geq 1$, but not for $c<1$. On the other hand, it was shown by Cramér, see Zygmund, $\left[24\right.$, p. 110], that there exists a critical value $0<c_{\text {crit }}<1$ such that a Gibbs phenomenon takes place for $c>c_{\text {crit }}$ but not for $c<c_{\text {crit }}$. The value of $c_{\text {crit }}$ has been calculated with three-digit accuracy by Gronwall, but Otto Szász had doubts about the correctness of the third digit. At Szász' urging Loewner wrote a program for the Mark II to test for the Gibbs phenomenon, and found that, indeed, the third digit of the published value of $c_{\text {crit }}$ was incorrect. How Szász knew this remains a mystery.

Received November 19, 1997.

1991 Mathematics Subject Classification. Primary 01, 35, 39, 76. 
The Gibbs phenomenon is not only of theoretical but also of practical importance; the overshoot introduces serious errors into any straightforward calculation by Fourier series of discontinuous functions, such as occur in the description of flows containing shocks. Using arithmetic means of Fourier series does not help, for this introduces serious errors of its own. It is worth mentioning that recently David Gottlieb succeeded in eliminating the Gibbs phenomenon in spectral calculations without sacrificing overall accuracy.

The Mark II was digital and used mechanical relays; there were in those distant days other rudimentary computers available. There was Vannevar Bush's Differential Analyzer, an analogue device, and Eckert and Mauchly's ENIAC, the first electronic computer. In addition, many laboratories including Los Alamos and the Ballistic Research Laboratory at Aberdeen had figured out how to use punch card sorters to perform arithmetic operations. It was on just such a contrivance that von Neumann first tested his ideas on computational fluid dynamics. While working at Los Alamos during WW II, von Neumann came across the problem of computing compressible flows that contain strong shocks. He soon came to the conclusion that this can be accomplished only by numerical computations. He responded to this challenge with his usual vigor: he proceeded to invent computational fluid dynamics, programmable electronic digital computers, computer architecture, programming, flow charts, and the stability and error analysis of various numerical schemes. He was keenly aware that computing was not just for designing bombs, but that it would revolutionize all of the exact sciences as a tool for theorists to obtain numerical answers, and also to use for exploration in the manner of experimentalists. His visionary words, delivered at a meeting in Montreal in 1945, are still worth quoting:

... really efficient high-speed computing devices may, in the field of nonlinear partial differential equations as well as in many other fields which are now difficult or entirely denied of access, provide us with those heuristic hints which are needed in all parts of mathematics for genuine progress.

The organization of this paper is as follows: Section 2 is an analysis of von Neumann's wartime work in computational fluid dynamics in the light of our present understanding; see Lax [12], and Hou and Lax [10]. Section 3 describes some striking later results and a conjecture by Holian and others on nonlinear vibrating systems that are connected with von Neumann's early work.

2. The problem von Neumann tackled was the calculation of the flow of compressible gas in the presence of shocks and contact discontinuities. In such a problem the path of the discontinuities must be tracked simultaneously with calculating the values of pressure, density, and velocity on either side of the discontinuity. Von Neumann had the brilliant idea of dispensing with shock tracking by treating shocks as rapid transition. This strategy has been dubbed shock capturing, and is used very widely.

The equations used by von Neumann were the Lagrangian form of the laws governing compressible flow in one space dimension:

$$
\begin{aligned}
& u_{t}+p_{x}=0, \\
& V_{t}-u_{x}=0,
\end{aligned}
$$




$$
e_{t}+p u_{x}=0 .
$$

Here $u$ is velocity, $p$ is pressure, $V$ is specific volume, and $e$ is specific internal energy. The equation of state expresses $e$ as a function of $p$ and $V$; the simplest relation is a $\gamma$-law:

$$
e=\frac{p V}{\gamma-1}
$$

in air $\gamma=1.4$. Equation (1) is the conservation of momentum, (2) is conservation of mass. Multiplying (1) by $u$ and adding it to (3) gives the law of conservation of energy:

$$
\left(e+\frac{u^{2}}{2}\right)_{t}+(u p)_{x}=0 .
$$

Multiplying (2) by $p$ and adding it to (3) yields

$$
e_{t}+p V_{t}=0
$$

an equation containing only thermodynamic variables; multiplying (6) by an integrating factor gives the law of conservation of entropy:

$$
S_{t}=0
$$

$S$ some function of $p$ and $V$. For an equation of state of the form (4), $S=p V^{\gamma}$. As already observed by Riemann, a discontinuous solution can satisfy the conservation laws of mass, momentum, and energy in the integral sense. In modern language this can be expressed by saying that (1), (2), and (5) are satisfied in the sense of distribution. It is one of the basic results of compressible flow theory that the law of conservation of entropy is incompatible with the other three conservation laws for flows with shocks; it has to be replaced by the law of entropy increase across shocks.

For solving partial differential equations numerically the continuum equations have to be discretized. ${ }^{1}$ Von Neumann divided the relevant interval on the $x$-axis into a finite number of cells of equal size; he associated a call velocity $u_{k}$ with the center of each cell, and thermodynamic quantities $p_{k \pm 1 / 2}, V_{k \pm 1 / 2}$ with the endpoints of the cells. This made it possible to replace the space derivatives in Eqs. (1), (2), and (3) by centered difference quotients, accurate to second order. Von Neumann also introduced a staggered scheme in time that made it possible to replace the time derivatives with centered, second-order accurate difference quotients; for sake of simplicity we shall in this discussion leave time as a continuous variable. The semidiscrete version of von Neumann's scheme is the following system of ODEs, corresponding to Eqs. (1), (2), and (3):

$$
\begin{gathered}
\frac{d}{d t} u_{k}+\left(p_{k+1 / 2}-p_{k-1 / 2}\right) / \Delta=0, \\
\frac{d}{d t} V_{k+1 / 2}-\left(u_{k+1}-u_{k}\right) / \Delta=0, \\
\frac{d}{d t} e_{k+1 / 2}+p_{k+1 / 2}\left(u_{k+1}-u_{k}\right) / \Delta=0,
\end{gathered}
$$

\footnotetext{
${ }^{1}$ Von Neumann regarded the discretisation of space as natural, because the continuum theory of fluids is an idealisation of kinetic theory.
} 
with equation of state as before:

$$
e_{k+1 / 2}=f\left(p_{k+1 / 2}, V_{k+1 / 2}\right) .
$$

Here $\Delta$ is the size of a cell. Just as we did for the continuum equations, we can derive a law of conservation of energy by multiplying (7) by $u_{k}$ and adding it to (9):

$$
\left.\frac{d}{d t}\left(\frac{1}{2} u_{k}^{2}+e_{k+1 / 2}\right)+p_{k+1 / 2} u_{k+1}-p_{k-1 / 2} u_{k}\right) / \Delta=0
$$

Similarly we can derive a law of conservation of entropy by multiplying (8) by $p_{k+1 / 2}$ and adding it to (9). We get

$$
p_{k+1 / 2} \frac{d}{d t} V_{k+1 / 2}+\frac{d}{d t} e_{k+1 / 2}=0
$$

which, after multiplication by an integrating factor, becomes

$$
\frac{d}{d t} S\left(p_{k+1 / 2}, V_{k+1 / 2}\right)=0 .
$$

Actually, von Neumann did not solve the full system (1), (2), (3) of fluid dynamics. He omitted Eq. (3) and made the simplifying thermodynamical assumption that pressure is a function of the specific volume alone:

$$
p=p(V) \text {. }
$$

The test problem he chose, called today the von Neumann shock problem, was the motion of gas in a tube, one of whose ends is open, the other closed, in a uniform state initially, and moving with constant velocity toward the closed end;

$$
u_{k}(0)=a, \quad V_{k}(0)=W, \quad k>0 .
$$

If we take the closed end on the left, the velocity $a$ is negative. The closed end is described by

$$
u_{0}(t)=0, \quad t \geq 0 \text {. }
$$

The nature of the ensuing flow is easy to analyze. At the closed end a reflected shock wave appears instantaneously, propagating to the right, leaving behind a compressed zone where the gas is at rest. At the right endpoint a centered rarefaction wave is generated. Eventually, the two waves start to interact.

When the calculations were performed, the results showed rapid oscillations of the quantities $u_{k}(t)$ behind the shock front. Rather than viewing this as an aberration, von Neumann remarked that a shock wave is an irreversible process that converts kinetic energy into internal energy. Kinetic energy per unit mass is $\frac{1}{2} \bar{u}^{2}$, where $\bar{u}$ is the average velocity of the gas particles; the difference between the sum of the kinetic energies of the $N$ individual particles and $\frac{N}{2} \bar{u}^{2}$ is the internal energy of the gas. From this von Neumann drew two conclusions:

a) The solutions of the discrete equations (6), (7), and (12) converge as $\Delta$ tends to zero, but only in the weak sense.

b) The weak limit satisfies the differential equations (1) and (2) in the integral sense. 
In the light of modern understanding, conclusion a) is correct, but conclusion b) is not. We start with an alternate explanation of the rapid oscillations of the solutions of the discrete equations. Multiply Eq. (7) by $u_{k}$, Eq. (8) by $p_{k+1 / 2}$, and subtract. We get

$$
u_{k} \frac{d}{d t} u_{k}-p_{k+1 / 2} \frac{d}{d t} V_{k+1 / 2}+\left(u_{k} p_{k+1 / 2}-u_{k} p_{k-1 / 2}+p_{k+1 / 2} u_{k+1}-p_{k+1 / 2} u_{k}\right) / \Delta=0 \text {. }
$$

We can rewrite this as

$$
\frac{d}{d t}\left(\frac{1}{2} u_{k}^{2}-P_{k+1 / 2}\right)+\frac{p_{k+1 / 2} u_{k+1}-p_{k+1 / 2} u_{k}}{\Delta}=0
$$

where

$$
P_{k+1 / 2}=P\left(V_{k+1 / 2}\right)
$$

and

$$
\frac{d P}{d V}=p(V)
$$

Equation (14) is a discrete conservation law, as are (7) and (8). Define the functions $u_{\Delta}(x, t)$ and $p_{\Delta}(x, t)$ to be equal to $u_{k}(t)$, respectively $p_{k+1 / 2}(t)$, in intervals of length $\Delta$ centered at $x=k \Delta$ and at $x=(k+1 / 2) \Delta$. We appeal now to the Lax-Wendroff theorem:

Suppose there is a sequence of values of $\Delta$ tending to zero such that $u_{\Delta}$ and $p_{\Delta}$ tend in the $L^{1} \cap L^{\infty}$ sense to limits $u$ and $p$. Then $u$ and $p$ satisfy, in the sense of distributions, the continuum limit of the discrete conservation laws.

Applying this to the discrete conservation laws (7), (8), and (14) we conclude that $u$ and $p$ defined above satisfy in the sense of distribution theory the conservation laws (1), (2) and the additional one from (14):

$$
\left(\frac{1}{2} u^{2}-P(V)\right)_{t}+(p u)_{x}=0 .
$$

However, according to the theory of hyperbolic systems of conservation laws, two functions $u$ and $p$ with discontinuities cannot satisfy three independent conservation laws. This seeming contradiction shows that no subsequence of $u_{\Delta}, p_{\Delta}$ converges to a limit in the $L^{1} \cap L^{\infty}$ topology. In other words, the set of functions $u_{\Delta}, p_{\Delta}$ is not compact in the $L^{1} \cap L^{\infty}$ topology. This is a very strong indication that the functions $u_{\Delta}, p_{\Delta}$ oscillate more and more as $\Delta$ tends to zero.

Suppose now that, as suggested by von Neumann, a sequence $u_{\Delta}, V_{\Delta}$, and $p_{\Delta}$ converges weakly to limits $\bar{u}, \bar{V}$, and $\bar{p}$. Then integration of the conservation laws still shows that $\bar{u}, \bar{V}$, and $\bar{p}$ satisfy (1) and (2):

$$
\begin{aligned}
& \bar{u}_{t}+\bar{p}_{x}=0, \\
& \bar{V}_{t}+\bar{u}_{x}=0,
\end{aligned}
$$

but it is no longer true that $p$ and $V$ are related by the equation of state (12). For if $p(V)$ is a nonlinear function,

$$
w-\lim p(V) \neq p(w-\lim V) .
$$


We call a difference scheme for a system of conservation laws dispersive if it implies an extra conservation law. The arguments presented above show that solutions of a dispersive scheme cannot converge strongly to solutions with shocks, and if they converge weakly, the weak limit fails to satisfy the original conservation laws.

We point out that the difference scheme (7), (8), (9) is dispersive, since it implies not three but four conservation laws. Therefore, their solutions suffer the same fate as solutions of the reduced system (7), (8), and (12).

Other striking examples of dispersive schemes are furnished by various discretizations of the scalar equation

$$
u_{t}+u u_{x}=0
$$

such as

$$
\frac{d}{d t} u_{k}+u_{k}\left(\frac{u_{k+1}-u_{k-1}}{2 \Delta}\right)=0
$$

studied by Goodman and Lax [5],

$$
\frac{d}{d t} u_{k}+\left(\frac{u_{k+1}+u_{k}+u_{k-1}}{3}\right)\left(\frac{u_{k+1}-u_{k-1}}{2 \Delta}\right)=0,
$$

studied by Levermore and Liu [15], and

$$
\frac{d}{d t} u_{k}+\frac{u_{k+1}^{2}-u_{k-1}^{2}}{2 \Delta}=0
$$

studied by Rosales and Turner [19]. All are dispersive; numerical experiments indicate oscillatory behavior and weak convergence to functions that do not satisfy the conservation form of the scalar equation.

A few years after his first effort, von Neumann gave up on dispersive schemes, but not on shock capturing. He and Richtmyer [18] devised a dissipative scheme, containing an artificial viscosity. It implied no additional conservation law; in fact, it was not obvious that it implies the law of conservation of energy. That it does was shown only ten years later by an elegant argument of Trulio and Trigger [22].

3. Von Neumann noticed an interesting connection between the lattice vibrations and the Lagrange equations of motion with the simplified thermodynamics (12). The connection is furnished by the Euler coordinates $X(x, t)$; recall that the Euler coordinates move with flow speed:

$$
X_{t}=u \text {. }
$$

Setting this into Eq. (2) we get that

$$
V_{t}-u_{x}=V_{t}-X_{t x}=\left(V-X_{x}\right)_{t}=0 .
$$

Consequently, if $V=X_{x}$ holds at $t=0$, it holds for all time. Setting this into Eq. (1) gives the nonlinear wave equation

$$
X_{t t}+p\left(X_{x}\right)_{x}=0
$$


One can proceed similarly with Eqs. (7) and (8). Define $X_{k}$ analogously by

$$
\frac{d}{d t} X_{k}=u_{k}
$$

Setting this into (8) gives

$$
\frac{d}{d t}\left(V_{k+1 / 2}-\left(X_{k+1}-X_{k}\right) / \Delta\right)=0 .
$$

It follows that if $X_{k}(0)$ is so chosen that

$$
V_{k+1 / 2}=\frac{X_{k+1}-X_{k}}{\Delta}
$$

holds at $t=0$, then it holds for all $t$. Setting this into (7) we get, using (12), that

$$
\Delta \frac{d^{2}}{d t^{2}} X_{k}+p\left(\frac{X_{k+1}-X_{k}}{\Delta}\right)-p\left(\frac{X_{k}-X_{k-1}}{\Delta}\right)=0 \text {. }
$$

Rescaling:

$$
X_{k}=\Delta q_{k}, \quad t=\Delta s
$$

turns our equation into the form

$$
\frac{d^{2}}{d s^{2}} q_{k}+p\left(q_{k+1}-q_{k}\right)-p\left(q_{k}-q_{k-1}\right)=0
$$

As von Neumann noted, Eq. (16) describes the vibrations of a one-dimensional lattice consisting of unit masses, each linked to its two neighbors by identical nonlinear springs. The function $p(d)$ that in the simplified equation of state (12) relates pressure to specific volume appears here as the dependence of the force exerted by the spring on displacement.

In the seventies Holian and Straub [8] started a systematic study of the shock problem for lattice vibrations:

$$
q_{k}(0)=k, \quad \dot{q}_{k}(0)=-a, \quad k>0, \quad q_{0}(t)=0 .
$$

They experimented with various molecular force laws, such as Leonard-Jones, Morse and Toda; they were unaware - as was everyone else - of von Neumann's study of forces that obey a $\gamma$-law. Their findings were consistent with those of von Neumann, but went considerably beyond them. They observed the emergence of shock waves from the fixed site $k=0$, and noted that the $q_{k}$ perform large scale oscillations behind the shock. They discovered many details about these oscillations:

i) The oscillations are, roughly, on mesh scale.

ii) There is a critical shock strength, as measured by the initial velocity $a$ imparted to the masses, with the following property: for shock strength less than critical there is a region adjacent to $k=0$ expanding with some speed less than shock speed, that is, free of oscillations. For shock strength greater than critical the motion is oscillatory everywhere behind the shock; but there is an expanding region adjacent to $k=0$ where the oscillation is periodic and binary, i.e., satisfies $q_{k}(t)=q_{k+1}(-t)$. The numerical evidence is marshalled in Hou and Lax [10].

In a subsequent paper [9], Holian, Flaschka, and McLaughlin examined the Toda lattice:

$$
p(d)=e^{-d}
$$


making use of its complete integrability, they were able to determine the critical value of shock strength, and to prove that it has the properties listed under ii) above. More recently Venakides, Deift, and Oba [23], using complete integrability, have given a very detailed description of the shock problem. This work was completed by Kamvissis [11].

Vibrations of the Toda lattice with more general initial conditions were studied by McLaughlin and Deift [2]; their results are getting near to proving von Neumann's more than 50-year-old conjecture about weak convergence stated in Sec. 2.

All these impressive proofs, and related ones concerning other dispersive approximations, suffer from a severe limitation: they work only for completely integrable systems. On the other hand, the numerical evidence is overwhelming that the phenomena described here: shock formation, critical shock strength, weak convergence, take place also for all force functions $p(d)$, regardless of integrability. Yet at present we have not the faintest idea how to go about proving such results. I recommend these challenging problems to the attention of young analysts.

This work was supported by the Applied Mathematical Sciences Program of the U.S. Department of Energy under contract DE-FG02-88ER25053.

\section{REFERENCES}

[1] H. Cramér, Études sur la sommation des series de Fourier, Arkiv för Matematik 13, \#20 (1919)

[2] P. Deift and K. McLaughlin, A continuum limit of the Toda lattice, Memoirs Amer. Math. Soc. 131, no. 624 (1988)

[3] H. Flaschka, On the Toda lattice. II. Inverse-scattering solution, Progr. Theoret. Phys. 51, 703-716 (1974)

[4] E. Fermi, J. Pasta, and S. Ulam, Studies on nonlinear problems I, Nonlinear Wave Motion, Lectures in Appl. Math., vol. 15, Amer. Math. Soc., Providence, RI, 1974

[5] J. Goodman and P. D. Lax, On dispersive difference schemes I, Comm. Pure Appl. Math. 41, 591-613 (1988)

[6] D. Gottlieb and C.-W. Shu, On the Gibbs phenomenon and its resolution, SIAM Review 39, 644668 (1997)

[7] B. Hayes, Binary modulated oscillations in a semi-discrete version of Burgers equation, Physica D 106, 287-313 (1997)

[8] B. L. Holian and G. K. Straub, Molecular dynamics of shock waves in one-dimensional chains, Phys. Rev. B 18, 1593-1608 (1978)

[9] B. L. Holian, H. Flaschka, and K. McLaughlin, Shock waves in the Toda lattice: Analysis, Phys. Rev. A 24, 2595-2623 (1981)

[10] T. Hou and P. D. Lax, Dispersive approximations in fluid dynamics, Comm. Pure Appl. Math. 44, 1-40 (1991)

[11] S. Kamvissis, On the Toda shock problem, Physica D 65, 242-266 (1993)

[12] P. D. Lax, On Dispersive Difference Schemes, Physica 18D, North-Holland, Amsterdam, 1986, pp. 250-254

[13] P. D. Lax and B. Wendroff, Systems of conservation laws, Comm. Pure Appl. Math. 13, 217-237 (1960)

[14] P. D. Lax and D. Levermore, The small dispersion limit of the Korteweg-de Vries equation, Comm. Pure Appl. Math., I: pp. 253-290, II: pp. 571-593, III: pp. 160-169 (1983)

[15] C. D. Levermore and J.-G. Liu, Large oscillations arising in a dispersive numerical scheme, Physica D 99, 191-216 (1996)

[16] J. von Neumann, Proposal and analysis of a new numerical method in the treatment of hydrodynamical shock problems, Collected Works, vol. 6, Pergamon Press, London, 1963

[17] J. von Neumann and H. H. Goldstine, On the principles of large scale computing machines, Collected Works, vol. 5, Pergamon Press, London, 1963

[18] J. von Neumann and R. D. Richtmyer, A method for the numerical calculation of hydrodynamic shocks, J. Appl. Phys. 21, 232-237 (1950) 
[19] R. R. Rosales and C. V. Turner, The small dispersion limit for a nonlinear semidiscrete system of equations, Stud. Appl. Math. 99, 205-254 (1997)

[20] O. Szász, Gibbs phenomenon for Hausdorff means, Trans. Amer. Math. Soc. 69, 1077-1093 (1950)

[21] M. Toda, Theory of Nonlinear Lattices, Springer-Verlag, Berlin-New York, 1981

[22] J. G. Trulio and K. R. Trigger, Numerical Solution of One Dimensional Hydrodynamical Shock Problems, UCRL Report 6522, 1961

[23] S. Venakides, P. Deift, and R. Oba, The Toda shock problem, Comm. Pure Appl. Math. 44, 11711242 (1991)

[24] A. Zygmund, Trigonometric Series, second edition, Cambridge University Press, Cambridge, 1959 